\title{
Solid noninfectious growing masses over cerebrospinal fluid shunts: report of 3 cases
}

\author{
Hector E. James, MD, ${ }^{1}$ Richard A. Postlethwait, PA-C,' and E. Dayan Sandler, MD² \\ 1Division of Pediatric Neurosurgery, Department of Neurosurgery, University of Florida College of Medicine Jacksonville/Wolfson \\ Children's Hospital; and 2Department of Pathology, Wolfson Children's Hospital/Baptist Medical Center, Jacksonville, Florida
}

\begin{abstract}
The authors describe 3 children who presented with progressively enlarging skin-covered solid masses over the shunt catheter in the neck/clavicular region. The authors reviewed the clinical, laboratory, pathological, radiographic, and follow-up data for all 3 patients and reviewed the literature on the subject. The patients had no clinical evidence of an infectious process. Surgical exploration revealed that masses were surrounding and encasing the shunt tubing to which they were strongly attached. Pathological studies of the tissues demonstrated varying degrees of exuberant chronically inflamed granulation tissues, interstitial fibrosis, and dystrophic calcification. One patient had associated thinning of the skin overlying the mass and subsequently developed ulceration. No infectious organisms were observed. The cerebrospinal fluid aspirates from the shunts did not yield any organisms. There has been no recurrence of the masses. The presence of a growing mass over the shunt tube in the neck or the chest region without clinical evidence of infection does not indicate that the mass should be treated with antibiotics and complete shunt removal. Rather, the mass can be cured by extirpation and with "bypass" new shunt tubing locally.
\end{abstract}

http://thejns.org/doi/abs/10.3171/2014.10.PEDS14237

KEY WORDS CSF shunts; aseptic solid masses; chronic inflammation; hydrocephalus

$\mathrm{T}$ ISSUE reaction around CSF shunts may be a sign of bacterial shunt infection. ${ }^{3,4,7}$ CSF shunt infections are most commonly treated with a combination of antibiotic therapy and shunt removal. $3,4,7$ When the infection is cured, another surgical intervention is required to insert a new shunt. ${ }^{3,4,7}$ These procedures may require prolonged hospitalization., ${ }^{3,4,7}$

We report on 3 patients who presented with indolent, slowly progressive growing masses over the shunt tubing in the neck and/or clavicular region, without evidence of associated infections. The patients were treated by excision of the mass and removal of the shunt tubing, and insertion of a "bypass" tubing and reanastomosis to the shunt in a region contiguous to the previous shunt track. This technique resolved the issue in all 3 patients, and none of the masses recurred.

\section{Case Reports}

As part of an ongoing review of the long-term management and outcomes of pediatric neurosurgery patients being followed in the multidisciplinary neurosciences clinic, 3 patients were identified who presented with indolent pro- gressive growth of subcutaneous masses over their ventriculoperitoneal shunt tracks. The charts and pertinent medical information are part of this report. Authorization for the chart review was obtained from the University of Florida Health Jacksonville Institutional Review Board.

\section{Case 1}

This 9-year-old boy presented with an enlarging mass overlying the clavicle, lower neck, and upper chest in the region of the shunt track. The patient was otherwise well, active, and attending school, with no reported headaches and he was free of signs or symptoms of shunt malfunction and infection. His medical history revealed that he was born prematurely and acquired posthemorrhagic progressive hydrocephalus. At 3 months of age he had undergone insertion of a parietooccipital ventriculoperitoneal shunt. He had not required any shunt revisions. On examination, the skin was intact, and a mass had developed that measured $8 \times 10 \mathrm{~cm}$. There was no local inflammation, and the mass was nontender and felt solid to palpation. There was no evidence of lymphadenopathy in the cervical and axillary regions. Radiographic studies revealed shunt continuity and normal locations of the proximal and distal ends.

SUBMITTED May 9, 2014. ACCEPTED October 3, 2014.

INCLUDE WHEN CITING Published online January 30, 2015; DOI: 10.3171/2014.10.PEDS14237.

DISCLOSURE This work was supported in part by the Lucy Gooding Charitable Foundation Trust and the Baptist Foundation of Northeast Florida. Both of these are notfor-profit organizations. The authors report no conflict of interest concerning the materials or methods used in this study or the findings specified in this paper. 
Cerebrospinal fluid aspirated from the shunt reservoir had no evidence of inflammation, and cultures and Gram staining were negative. The mass continued to grow, and excision was recommended to determine the etiology of the process. The mass was solid and gritty and encased the tubing. The proximal stump of the shunt tubing above the mass was sectioned and CSF flowed freely from it. The shunt was clamped, and the normal distal shunt tubing was exposed below the mass and was sectioned. The mass was then removed and sent for culture and pathological review. A new section of shunt catheter was tunneled medial to the previous site, and it was inserted and connected to the proximal and distal ends of the peritoneal catheter. The area was irrigated and sutured closed. The wounds healed well, there was no recurrent soft-tissue growth, and no shunt operations were performed in the 4 years of follow-up. The cultures of the CSF and tissues did not yield any organisms. Histological examination of the mass showed abundant dystrophic microcalcifications, fibrosis, and granulation tissue reaction (Fig. 1).

\section{Case 2}

This 7-year-old boy presented with a growing mass in the lower neck, clavicular, and upper chest region overlying the ventriculoperitoneal shunt track. The patient had no signs of infection, headaches, or symptoms of shunt malfunction. He was behaving normally both at home and at school. His medical history revealed that he was born prematurely and acquired posthemorrhagic hydrocephalus. He had undergone parietooccipital ventriculoperitoneal shunt insertion at 2 months of age. He never required shunt revision. On examination, a mass measuring $5 \times 10$ $\mathrm{cm}$ was present. The mass was nontender and felt solid to palpation. The skin was normal, and there were no inflammatory signs. The patient was treated in a similar fashion to the first patient. At surgery the mass was found to be encasing the shunt tubing. It was solid but not gritty. A bypass procedure was performed that was similar to that

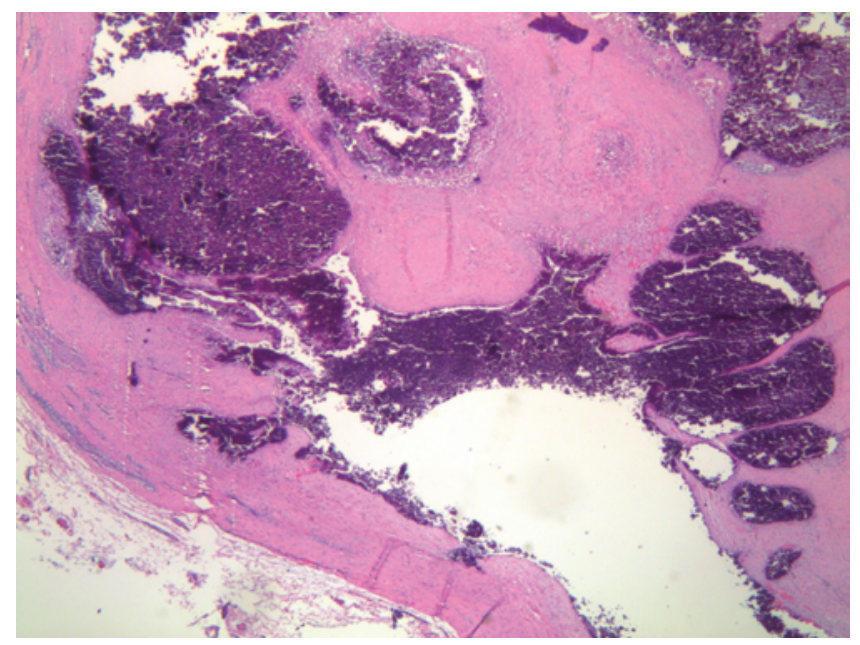

FIG. 1. Case 1. Histology of the mass. Surrounding the lumen created by the shunt catheter there is exuberant chronic granulation tissue reaction with microcalcifications. $H \& E$, original magnification $\times 50$. Figure is available in color online only. described in Case 1. The wound healed well. In 3 years of subsequent follow-up there has been no recurrence. Cultures of the CSF from the shunt and the mass did not show any organisms. Histological examination revealed exuberant chronically inflamed granulation tissue and fibrosis (Fig. 2).

\section{Case 3}

This 6-year-old girl presented with a growing soft-tissue mass overlying the valve mechanism in the upper mastoid region and over the shunt track in the upper neck. The patient's parents denied any signs of infection, headaches, or any signs/symptoms of shunt malfunction. Her medical history revealed that she was born prematurely and acquired posthemorrhagic progressive hydrocephalus. A frontal ventriculoperitoneal shunt had been inserted when the patient was 3 months old and never required revision. Examination revealed normal skin overlying a mass, which was not tender and felt solid to palpation. There was no obvious lymphadenopathy in the region. The mass measured approximately $5 \times 4 \mathrm{~cm}$. The patient's mother chose conservative treatment. During subsequent weeks the mass increased in size to $5 \times 7 \mathrm{~cm}$, and the skin seemed to be thinning. The CSF shunt aspirate did not yield evidence of an inflammation or organisms. A local skin ulceration occurred over the dome of the mass, and the patient's mother agreed to surgical intervention. An operation similar to that of the first 2 cases was performed. The area of ulceration healed well. The patient has not experienced a recurrence during the 8 years of follow-up. Cultures of the CSF and of the mass did not yield any organisms. Histological examination revealed acute and chronic inflammation, granulation, and interstitial fibrosis (Fig. 3).

\section{Discussion}

All 3 patients had the presence of shunt material, location of a growing mass in the lower neck and/or over the

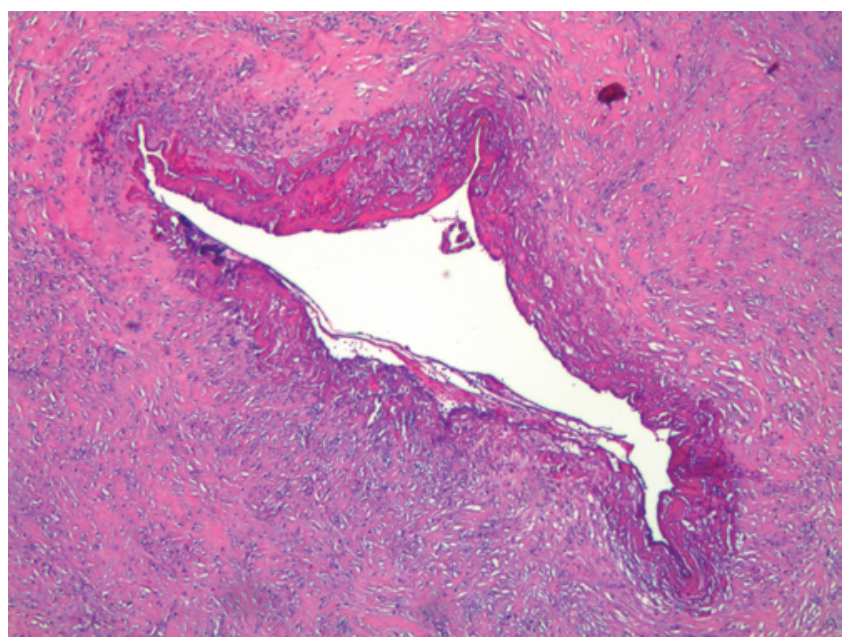

FIG. 2. Case 2. Histology of the mass. A lumen, which was created by the catheter, and is in the center of the image, is surrounded by a fibroblastic granulation tissue response. A condensation of collagen surrounds the lumen. A few chronic lymphohistiocytic cells are present. $H$ \& $E$, original magnification $\times 50$. Figure is available in color online only. 


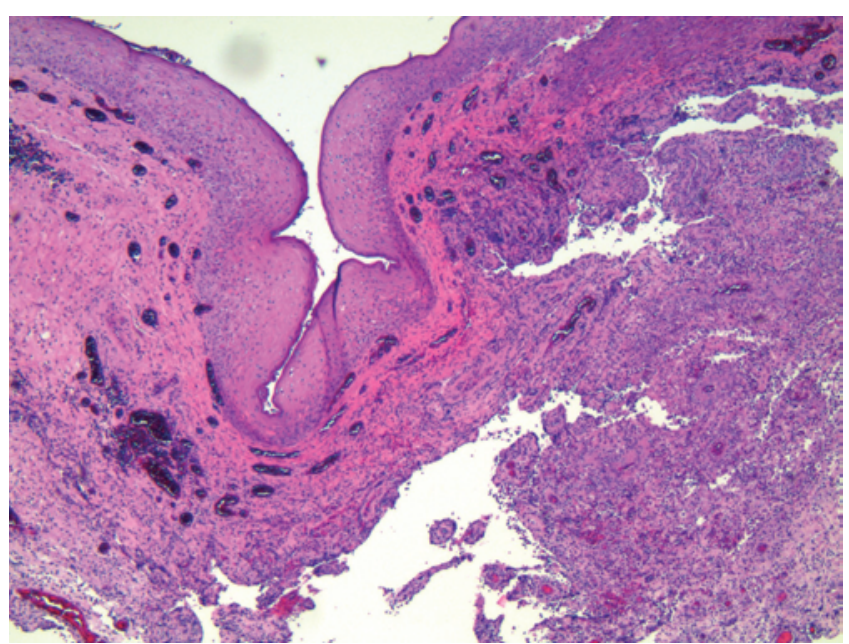

FIG. 3. Case 3. Histology of the mass reveals acute and chronic inflamed granulation tissue present beneath the skin. Eventually the inflammation led to breakdown of the overlying skin. $\mathrm{H} \& \mathrm{E}$, original magnification $\times 50$. Figure is available in color online only.

clavicle and upper chest, and a prolonged period between shunt implantation and presentation of the mass (Table 1). In addition, the CSF aspirates from all 3 shunts prior to operation and at the time of operation yielded neither inflammatory cells nor organisms. CSF cultures were monitored for 2 weeks before they were discarded. All 3 shunts were from different designs and manufacturers. All 3 original shunt insertions had taken place in different hospitals. None of the patients had signs or symptoms of shunt malfunction.

The histological studies of the masses revealed a chronic inflammatory response in all 3 patients, with varying degrees of exuberance, and 1 showed dystrophic microcalcifications (Table 2). No bacterial or fungal organisms were identified. The patient with a prolonged period of observation prior to surgical intervention (Case 3) developed thinning and subsequent breakdown of the skin overlying the mass. Histological examination revealed acute inflammatory changes in the area underlying the skin breakdown, with chronic inflammatory changes and a granulation tissue response.

Patients with ventriculoperitoneal shunts may present with enlarging soft-tissue masses over the chest wall, breast, or clavicular regions, due to shunt malfunction. This may occur with distal shunt obstruction and intraabdominal pseudocyst formation, or fracture of the shunt. ${ }^{2,6}$ The CSF may dissect and track around the shunt from the

TABLE 1. Clinical findings in 3 patients with solid noninfectious growing masses over cerebrospinal fluid shunts

\begin{tabular}{ccccc}
\hline $\begin{array}{c}\text { Case } \\
\text { No. }\end{array}$ & $\begin{array}{c}\text { Age } \\
\text { (yrs) }\end{array}$ & $\begin{array}{c}\text { Tmplantation to Mass } \\
\text { Presentation }(\mathrm{yrs})\end{array}$ & Location & $\begin{array}{c}\text { Mass } \\
\text { Size }(\mathrm{cm})\end{array}$ \\
\hline 1 & 9 & 9 & Neck/upper chest & $8 \times 10$ \\
\hline 2 & 7 & 6 & Neck/upper chest & $5 \times 10$ \\
\hline 3 & 6 & 6 & Mastoid/upper neck & $5 \times 7$ \\
\hline
\end{tabular}

TABLE 2. Findings from histopathological examination

\begin{tabular}{cccc}
\hline $\begin{array}{c}\text { Case } \\
\text { No. }\end{array}$ & $\begin{array}{c}\text { Granulation Tissue, Fibrosis, } \\
\text { Chronic Inflammation }\end{array}$ & Calcifications & $\begin{array}{c}\text { Skin } \\
\text { Ulcerations }\end{array}$ \\
\hline 1 & Yes & Yes & No \\
\hline 2 & Yes & No & No \\
\hline 3 & Yes & No & Yes \\
\hline
\end{tabular}

abdomen and present with a subcutaneous mass, due to progressive fluid accumulation. Most of these patients present with signs and symptoms of shunt malfunction and/ or abdominal distension. On palpation, these masses commonly have some degree of compressibility due to their fluid content. Jimenez et al. reported on 3 patients with reaction and skin breakdown overlying the ventriculoperitoneal shunt. ${ }^{5}$ The CSF was sterile in all 3 patients, and the histological studies of the surrounding tissues revealed acute and chronic inflammatory changes. Two of the patients had granulomas. Of interest, in all 3 patients the reaction was localized to the scalp directly over the reservoir and/or valve mechanism. All 3 patients had recurring skin breakdowns around the shunt, and subsequently they acquired bacterial infections. One patient was successfully treated with complete shunt removal. Another patient was shunt dependent, and, following successful treatment of the shunt infection with removal of the shunt, antibiotics, and insertion of a new shunt, required treatment with immunosuppression and prednisone to avoid reaction to the shunt. The third patient died following several shunt revisions and mobilization to the contralateral side. The authors postulated that the etiology of the process was an allergic localized reaction to the Silastic material of the shunt system. ${ }^{5}$

Implantation of solid silicone materials is followed by the formation of fibrous tissue with a minimal inflammatory response, forming a "pseudocapsule," which is commonly seen around shunt tracks. Other forms of reaction to silicone materials have been described. Winer et al. noted granuloma foreign-body reaction in the macrophages, histiocytes, epithelioid cells, lymphocytes, plasma cells, and giant multinucleated cells in the subcutaneous tissues following the injection of liquid silicone in humans. ${ }^{9}$ They coined the term "siliconoma" to describe this process and subsequently reproduced these changes following intraperitoneal silicone injections in mice. ${ }^{8}$ However, none of the reports indicate the presence of progressively enlarging masses that created such significant anatomical distortion as that which occurred in our 3 patients. We can speculate as to the mechanism causing this local soft-tissue reaction. One potential explanation for the presence of changes in the lower neck and upper chest regions may be because this area is submitted to repetitive traction and movement in the normal neck movements in day-to-day activity and/ or trauma. The sheath that is always seen around the shunt tubing may react to the constant friction with the shunt implant and, over a prolonged period of time, may exaggerate the chronic inflammatory and fibrous response. The paper by Anderson provides a detailed overview of the process of the biological responses to implanted materials. ${ }^{1} \mathrm{He}$ emphasized the "continuum" of the body's reaction to an 
implant: the local "injury" at the time of the implantation, inflammatory and wound healing responses, foreign body reactions, and fibrous encapsulation (scar formation). ${ }^{1}$ The initial response generates a provisional matrix formed at the implant site. Fibrin, the major component of the provisional matrix, plays a key role in the local neovascularization, which helps in the wound-healing process. The biocompatibility of Silastic is described in reference to the time and sequence of the capsule formation. Classically, the development of granulation tissue is considered to be part of chronic inflammation, but the presence of the $\mathrm{Si}$ lastic material induces varying degrees of granulation tissue reaction, which includes macrophages, fibroblasts, and capillary formation, and there is a difference from that of chronic inflammation without a foreign body. Granulation tissue is different from granulomas. In granulomas, there are small collections of macrophages and epithelioid cells (which are modified macrophages) and multinucleated giant cells. These giant cells are formed from the fusion of monocytes and macrophages, in an attempt to phagocytize foreign material. Anderson also emphasized that the reaction varies according to the form, topography, and size of the implant. ${ }^{1}$ If there is a persistent reactive inflammatory response to the implant, chronic inflammation will continue and the soft tissue may enlarge. He pointed out that motion in the implant site by the biomaterial may also lead to chronic inflammation. ${ }^{1}$

The vast majority of patients with CSF shunts do not develop enlarging soft-tissue masses, so there must be a unique element in patients who develop these changes. Although we cannot provide a precise logical explanation, we feel the matter deserves further investigation. We hope that by reporting these findings, we create an awareness of this condition and lead others to identify similar patients, which, in turn, helps clarify the etiology of this disease process.

\section{Conclusions}

Neurosurgeons need to be aware that the presence of a growing mass around the shunt tubing in the neck or upper chest region, without evidence of infection, does not always indicate a need for complete shunt removal and antibiotics. Parents and caregivers need to be informed of the slowly progressive nature of the process and that it might be cured by mass extirpation with "bypass" shunt tubing, as was performed in the patients reported on in this series.

\section{Acknowledgment}

We wish to acknowledge the excellent work of Mrs. Jennifer Santarone in the preparation and electronic submission of the manuscript.

\section{References}

1. Anderson JM: Biological responses to materials. Annu Rev Mater Res 31:81-110, 2001

2. Iyer HP, Jacob LP, Chaudhry NA: Breast cerebrospinal fluid pseudocyst. Plast Reconstr Surg 118:87e-89e, 2006

3. James HE, Bradley JS: Aggressive management of shunt infection: combined intravenous and intraventricular antibiotic therapy for twelve or less days. Pediatr Neurosurg 44:104111,2008

4. James HE, Bradley JS: Management of complicated shunt infections: a clinical report. J Neurosurg Pediatr 1:223-228, 2008

5. Jimenez DF, Keating R, Goodrich JT: Silicone allergy in ventriculoperitoneal shunts. Childs Nerv Syst 10:59-63, 1994

6. Kalra N, Mani NB, Jain M, Sidhu R, Gulati M, Suri S: Cerebrospinal fluid pseudocyst of the breast. Australas Radiol 46:76-79, 2002

7. Murphy K, Bradley J, James HE: The treatment of Candida albicans shunt infections. Childs Nerv Syst 16:4-7, 2000

8. Rees TD, Ballantyne DL Jr, Seidman I, Hawthorne GA: Visceral response to subcutaneous and intraperitoneal injections of silicone in mice. Plast Reconstr Surg 39:402-410, 1967

9. Winer LH, Sternberg TH, Lehman R, Ashley FL: Tissue reactions to injected silicone liquids: a report of three cases. Arch Dermatol 90:588-593, 1964

\section{Author Contributions}

Conception and design: James. Acquisition of data: Postlethwait, Sandler. Drafting the article: James, Sandler. Critically revising the article: Sandler. Reviewed submitted version of manuscript: all authors. Approved the final version of the manuscript on behalf of all authors: James.

\section{Correspondence}

Hector E. James, Lucy Gooding Pediatric Neurosurgery, 836 Prudential Dr., Ste. 1205 Pavilion Building, Jacksonville, FL 32207. email: hector.james@jax.ufl.edu. 